\title{
Hybridization of Fano and Vibrational Resonances in Surface-Enhanced Infrared Absorption Spectroscopy of Streptavidin Monolayers on Metamaterial Substrates
}

\author{
Kamil Boratay Alici, Member, IEEE
}

\begin{abstract}
We present spectral hybridization of organic and inorganic resonant materials and related bio-sensing mechanism. We utilized a bound protein (streptavidin) and a Fano-resonant metasurface to illustrate the concept. The technique allows us to investigate the vibrational modes of the streptavidin and how they couple to the underlying metasurface. This optical, label-free, nonperturbative technique is supported by a coupled mode-theory analysis that provides information on the structure and orientation of bound proteins. We can also simultaneously monitor the binding of analytes to the surface through monitoring the shift of the metasurface resonance. All of this data opens up interesting opportunities for applications in biosensing, molecular electronics and proteomics.
\end{abstract}

Index Terms-Avidin, biosensor, biotin, collective excitation, enhancement factor, fano resonance, metamaterial, microscopy, monolayer, nanoantenna, near-field effects, self-assembly, spectroscopy, streptavidin, surface-enhanced infrared absorption (SEIRA), surface plasmon, temporal-coupled mode theory, thin films.

\section{INTRODUCTION}

D ESIGN and development of an artificial substrate which provides a high signal-to-noise ratio (SNR) in Fourier transform infrared spectroscopy (FTIR) with high spectral selectivity and sensitivity can provide information about the structure, orientation, and ultimately biological function of various analytes such as peptides and proteins. This information is useful for detection of biological agents, diagnostic and monitoring of diseases, and drug discovery [1]. Ligand receptor-binding [2] and protein adsorption on self-assembled monolayers [3] are types

Manuscript received March 11, 2013; revised August 12, 2013; accepted December 1, 2013. Date of publication January 2, 2014; date of current version March 6, 2014. This work was supported in part by the Office of Naval Research under Grant N00014-10-1-0929), in part by the Air Force Office of Scientific Research under Grant FA8650-090-D-5037, in part by the National Science Foundation under Grant CMMI-0928664, and in part by the Welch Foundation under Grant F-1699. The review of this paper was arranged by Associate Editor Y.-H. Cho.

The author was with the University of Texas at Austin, Austin, TX 78712 USA. He is now with the Nanotechnology Research Center, Bilkent University, Ankara 06800, Turkey (e-mail: alici@bilkent.edu.tr).

This paper contains supplemental material available online at http:// ieeexplore.ieee.org (file size: $23 \mathrm{~KB}$ ).

Color versions of one or more of the figures in this paper are available online at http://ieeexplore.ieee.org.

Digital Object Identifier 10.1109/TNANO.2013.2296896 of analyte-surface binding interactions that have been proven to be practical to create monolayers of proteins on noble metal thin films. One of the most important and well-characterized proteins for fundamental studies of biorecognition is streptavidin [4], which is routinely used for the construction of supra-molecular systems by binding other functional units such as antibodies, colloids, inhibitors, oligonucleotides, and nucleic acids [5], [6]. Streptavidin and its ligand biotin are very stable and commercially available analytes whose highly specific interaction has a high binding constant $\left(K_{a}=10^{13} \mathrm{M}^{-1}\right)$ [4], which makes them a popular and well-established system for biosensing [7], [8]. This system has been widely used in the development of surface plasmon resonance (SPR) biosensors, which rely on the response of surface plasmons to changes in local refractive index [5], [6], [8]-[12]. SPR and LSPR (localized SPR) biosensors have been demonstrated and are promising for applications in various biomolecular systems [13], [14].

Utilization of surface plasmons [15] in order to increase the SNR of fluorescence [16], Raman [17]-[23] and infrared spectroscopy [19], [21], [23]-[31] has been studied extensively. A substrate involving a two-dimensional array of nanoshells provided significant enhancement to both surface-enhanced Raman spectroscopy (SERS) and surface-enhanced infrared absorption (SEIRA) by combining them effectively [19], [32]. Various types of periodically arranged hole and antenna arrays [21], [23]-[25], [27]-[29], [33] and metamaterial inspired designs [34]-[38] have been used in biosensing studies as SEIRA substrates. Resonant near-field enhancement in these substrates leads to increased SNRs for FTIR spectroscopy with highly selective frequency dependence. The operation frequency of such artificial structures depends on the geometry, content and period of the unit cell [39], [40], and can be designed to achieve strong coupling to vibrational modes of various analytes [38], [41], [42]. One can also obtain very sharp spectral features by using multiple nanoantennas within the unit cell [34], [43] as the coupling between the different modes of the nanoantennas create constructive or destructive interference in the far field yielding an ultrasensitive response.

Simultaneous detection of Protein A/G and Protein $\operatorname{IgG}$ monolayer thickness and Amide-I/II vibrational modes by using a Fano-resonant metamaterial substrate has been previously demonstrated. The Protein A/G directly binds to the gold surface of metamaterial antennas through the cysteine group [44]. In this paper, we apply the technique to the biotin-streptavidin system 
and observe hybridization of the metamaterial Fano resonance with streptavidin's Amide-I vibrational mode for the first time. Besides, we have investigated the following four outcomes of the technique: 1) binding of protein to the surface can be detected, 2) protein monolayer thickness can be estimated, 3) sensitivity is higher at the Fano resonance, 4) signal from the vibrational lines increases as the Fano resonance approaches them. The organization of the paper is as follows: after overview of the experimental, numerical, and theoretical methods, we present the design parameters and near-field enhancement of the antisymmetric Fano-resonant metamaterial unit cell. AFM and FTIR characterization of the fabricated metamaterial arrays will be followed by demonstration of index shift in the presence of streptavidin monolayer. As the Fano resonance approaches the Amide-I mode of the streptavidin, the SNR gradually increases and Amide-I frequency shifts consistently for all experiments.

\section{OVERVIEW OF EXPERIMENTAL AND THEORETICAL METHODS}

The metamaterial unit cell was designed via numerical simulations by using a finite-element method (FEM) and finite integration (FI) solvers. Temporal-coupled mode theory was used to construct an analytical model of the system (protein monolayer on gold metamaterial surface) [44]-[47]. Polarized reflection spectra and field enhancements were calculated by using both numerical and analytical calculations. Metamaterial arrays with different scale factors were fabricated on quartz substrates by using standard e-beam lithography techniques. After "piranha" cleaning, metamaterial samples were incubated in a solution with an optimized concentration of HPDP-biotin. The final step was the immobilization of streptavidin on the biotin self-assembled monolayer (SAM). After each step, i.e, 1) "piranha” cleaning, 2) SAM deposition, and 3) streptavidin immobilization, we measured the reflectance spectra of the samples using an FTIR microscope.

\section{DESIGN ENHANCEMENT FACTOR AND SPECTRAL CHARACTERIZATION OF FANO-RESONANT METAMATERIAL SURFACE}

The metamaterial unit cell is composed of a gold nanostrip oriented in the $y$-direction and another L-shaped nanostrip located nearby as shown in Fig. 1(a). We have fabricated substrates that include four different resonator arrays each with an area of $250 \mu \mathrm{m}^{2}$. The atomic force microscopy (AFM) characterization was performed using an Agilent 5500AFM in tapping mode. A typical micrograph of the unit cell without any smoothing is shown in Fig. 1(a). Simulated reflection spectrum for normal incidence and angle averaged cases are shown in Fig. 1(b). Angle of incidence was spanned from $15^{\circ}$ to $35^{\circ}$ with $5^{\circ}$ steps and results were averaged to obtain the overall response. Electric field distribution, enhancement, and surface current distribution at the frequency of interest are shown in Fig. 1(c) and (d). IR measurements were performed using a Thermo Scientific Nicolet 6700 Spectrometer with $\mathrm{KBr}$ beam splitter. It is equipped with a Continuum Microscope with liquid $\mathrm{N}_{2}$ cooled (a)

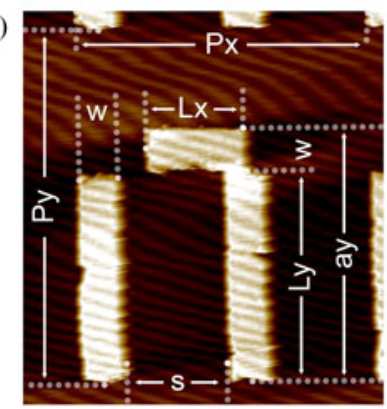

(c)

(d)
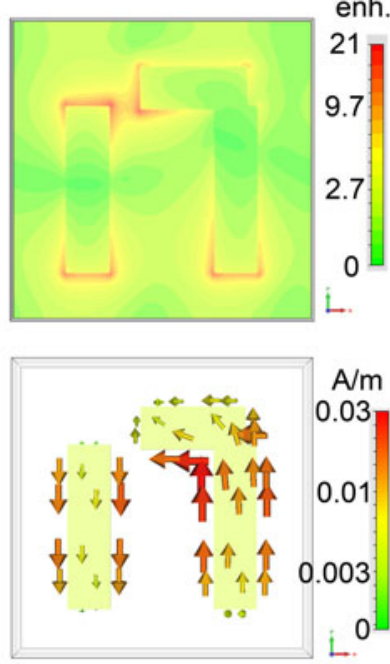

(f)

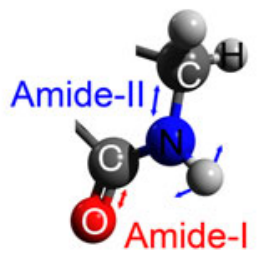

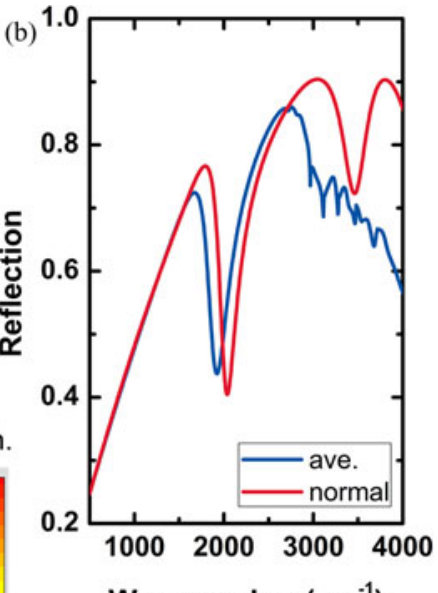

Wavenumber $\left(\mathrm{cm}^{-1}\right)$

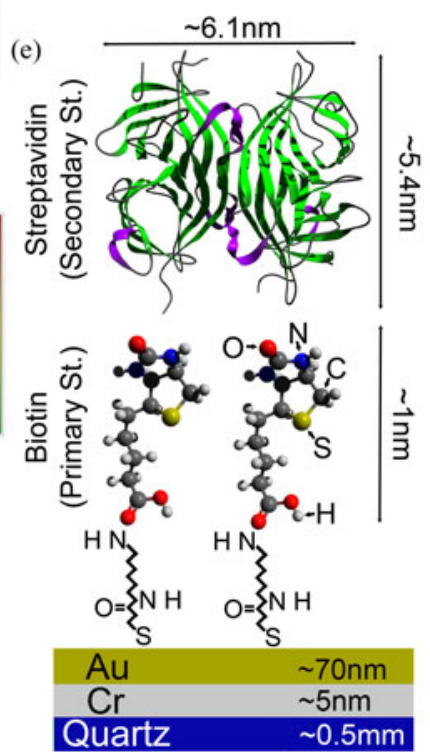

Fig. 1. (a) AFM topography data for a representative unit cell. The parameters of this representative metamaterial unit cell are as follows: the width of the strips is $w=0.30 \mu \mathrm{m}$, the lengths of the strips are $L_{x}=0.74 \mu \mathrm{m}$ in the $x$-direction, and $L_{y}=1.16 \mathrm{~nm}$ in the $y$-direction, the periods in the $x$-and $y$-directions are $P_{x}=2.04 \mu \mathrm{m}$ and $P_{y}=1.85 \mu \mathrm{m}$, the thickness of the gold layer is $h \sim 70 \mathrm{~nm}$, and the chromium adhesion layer between the gold layer and quartz substrate is approximately $5 \mathrm{~nm}$ thick. (b) FEM simulation results for the representative metamaterial unit cell: normal incidence and $25^{\circ}$ of incidence with $\pm 10^{\circ}$ of angle averaging. (c) Map of the electric field enhancement factor at the resonant frequency in the $x y$ plane. (d) Surface current density at the resonant frequency in the $x y$ plane. (e) Schematic of functionalized substrate (not to scale): primary structure of biotin and secondary structure of streptavidin are provided. (f) Amide molecule and its vibrational modes.

mercury cadmium telluride (MCT) detector and Parker Balston purge gas generator. The IR beam was focused with a $15 \times \mathrm{Re}-$ flachromat cassegrain objective and the physical aperture was set to $100 \mu \mathrm{m}^{2}$. The reflection was measured at an incidence angle of $25^{\circ}$ with $\pm 10^{\circ}$ angular divergence and the results are given as coaddition of 128 scans with $4 \mathrm{~cm}^{-1}$ resolution. The spectra were smoothed with a 19 point second-order SavitzkyGolay function after subtracting residual $\mathrm{H}_{2} \mathrm{O}$ vapor and $\mathrm{CO}_{2}$ lines. For an incident electromagnetic wave with the electric field polarized in the $y$-direction, the two lowest order modes (quadrupole and dipole) are of interest for generating a Fano 
resonance. The interference of a broadband dipole mode and a narrowband quadrupole mode generates an ultrasharp spectral feature at the Fano resonance. This ultrasharp resonance yields very large electric field enhancements [34] as shown in Fig. 1(c). The electric field intensity $5 \mathrm{~nm}$ above the gold surface shows an enhancement factor of about 25 at the Fano resonance.

\section{BiOTIN SAM USED AS A LINKER BETWEEN GOLD SURFACE AND STREPTAVIDIN MONOLAYER}

Because the resonator arrays are gold, oxidation of the metal surfaces was not a concern under ambient conditions; this is a great advantage of using gold [48]. Before functionalization, the resonator array substrate was cleaned with "piranha solution" (1:3 sulfuric acid: $30 \%$ hydrogen peroxide), which removes any organic contaminants from the surface [49], [50]. Then, the substrate was exposed to a $10 \mu \mathrm{M}$ solution of HPDP-biotin (EZLink biotin, Pierce Bio) which readily forms strong gold-sulfur bonds to the surface [51]. The biotinylated substrate was then exposed to a $10 \mu \mathrm{M}$ solution of streptavidin. Fig. 1(e) shows a schematic of the final functionalized surface, and Fig. 1(f) shows the strong Amide-I (C-O stretch) and Amide-II (C-N stretch and N-H bend) vibrational modes. The length of an HPDPbiotin molecule is $2.9 \mathrm{~nm}$, and the diameter of streptavidin is obtained as 5.4-6.1 nm from atomic resolution crystallographic study [52]. Therefore, the maximum total monolayer thickness is approximately $8.4-9.0 \mathrm{~nm}$ that also depends on the contact angle of biotin and streptavidin orientation. The structure of streptavidin is composed of four subunits each can bind to a biotin with $88 \mathrm{~kJ} / \mathrm{mol}$ bond energy [8], [53], [54]. Its molecular weight is approximately $60 \mathrm{kDa}$. Assuming maximum surface coverage and using the minimum diameter of streptavidin, we can estimate the maximum possible density of streptavidin molecules to be $4.9 \times 10^{4}$ molecules per square micrometer. It is not unusual to have defects in the biotin and streptavidin layers, so these estimates are upper limits only [55]-[57].

\section{SNR OF FTIR MiCROSPECTROSCOPY AT THE VIBRATIONAL LINES DEPENDS ON THE FANO-RESONANCE FREQUENCY OF METAMATERIAL SUBSTRATE}

The resonance frequencies of the metamaterial arrays strongly depend on the dielectric constant of the surrounding environment and red-shift in the presence of a protein monolayer. Fig. 2 shows reflectance FTIR spectra of a representative metamaterial substrate composed of four arrays with the unit cell parameters scaled in the $x y$ plane. We observed a consistent red-shift in the presence of a streptavidin monolayer for all four arrays and reproduced these results in two successful experiments on two samples (four datasets in total). In Fig. 2, we also show the results of temporal coupled mode theory (TCMT) developed for the specific metamaterial unit cell in combination with an approximate frequency shift calculation by using the first-order perturbation theory [58]. The expression reduces to double Lorenzian as follows: [44]

$$
R=\left|\frac{\alpha_{M}^{2}}{j\left(\omega-\omega_{M}\right)+\gamma_{M}}+\frac{\alpha_{E}^{2}}{j\left(\omega-\omega_{E}\right)+\gamma_{E}}\right|^{2}
$$

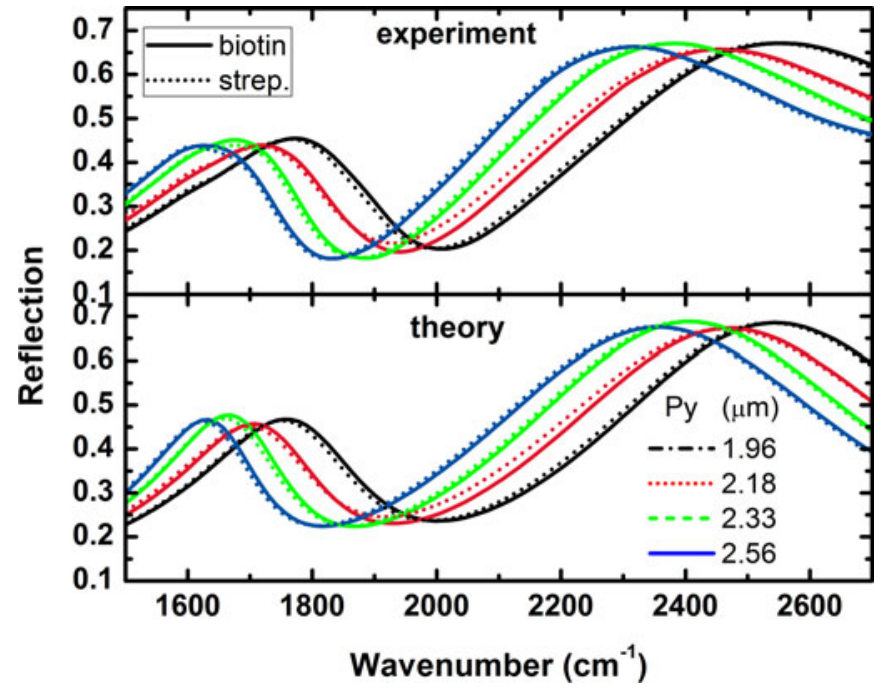

Fig. 2. (Top) Reflectance spectra from four resonator arrays with varying periodicities. (Bottom) Analytical model calculation results for the same array.

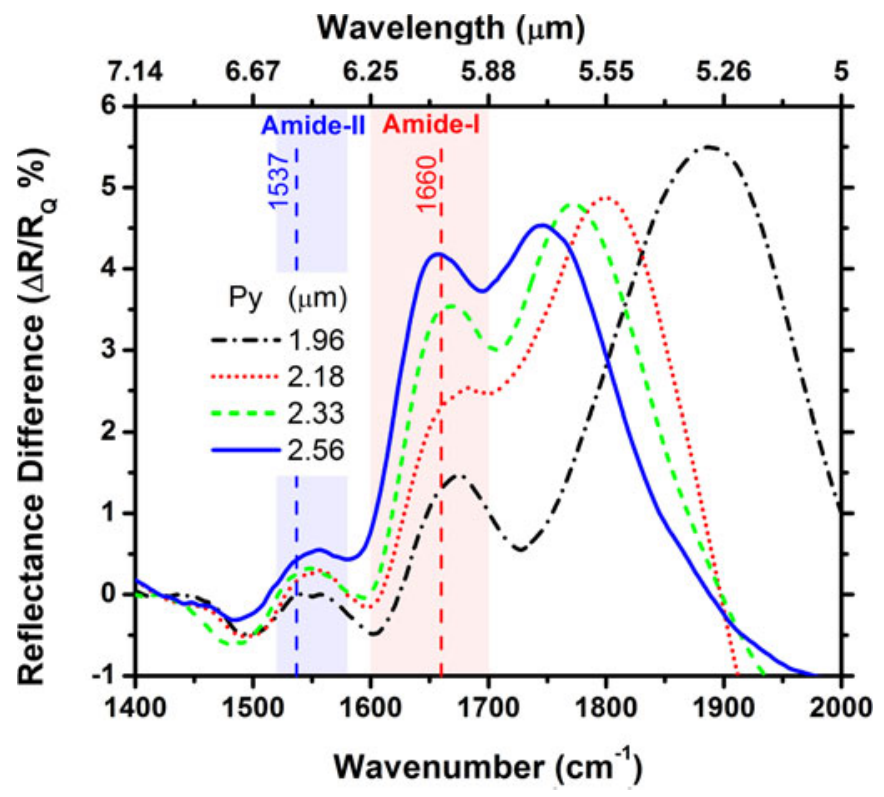

Fig. 3. Normalized experimental difference spectra of a representative sample. Position and denatured bands of the Amide-I/II are indicated with dashed lines and vertical bars, respectively. The difference peak is maximum at the Fano resonance.

where $\alpha_{M}$ and $\alpha_{E}$ coupling constants of the modes to the external source; $\omega_{M}$ and $\omega_{E}$ are eigenfrequencies of the modes with loss factors $\gamma_{M}$ and $\gamma_{E}$. In the presence of a thin dielectric layer with thickness $h$ the eigenfrequencies shift according to $\Delta \omega_{M} \sim-\left(\omega_{M} \Delta \varepsilon h\right) / l_{M}$, where $\Delta \varepsilon$ is the difference of the dielectric constants of the two media and $l_{M}$ is the surface averaged field localization length.

The spectral sensitivity of the resonator arrays is shown by the normalized reflectance difference spectra: $\Delta R / R_{Q}$, where $\Delta R=$ $R_{\mathrm{SAM}}-R_{\mathrm{PROTEIN}}$, and $R_{Q}$ is the reflectance at the quadrupole peak. In Fig. 3, we see the maximum of the difference spectra is at the Fano-resonance point, which implies the sensitivity to the index change is maximum at that point. Additionally, we 

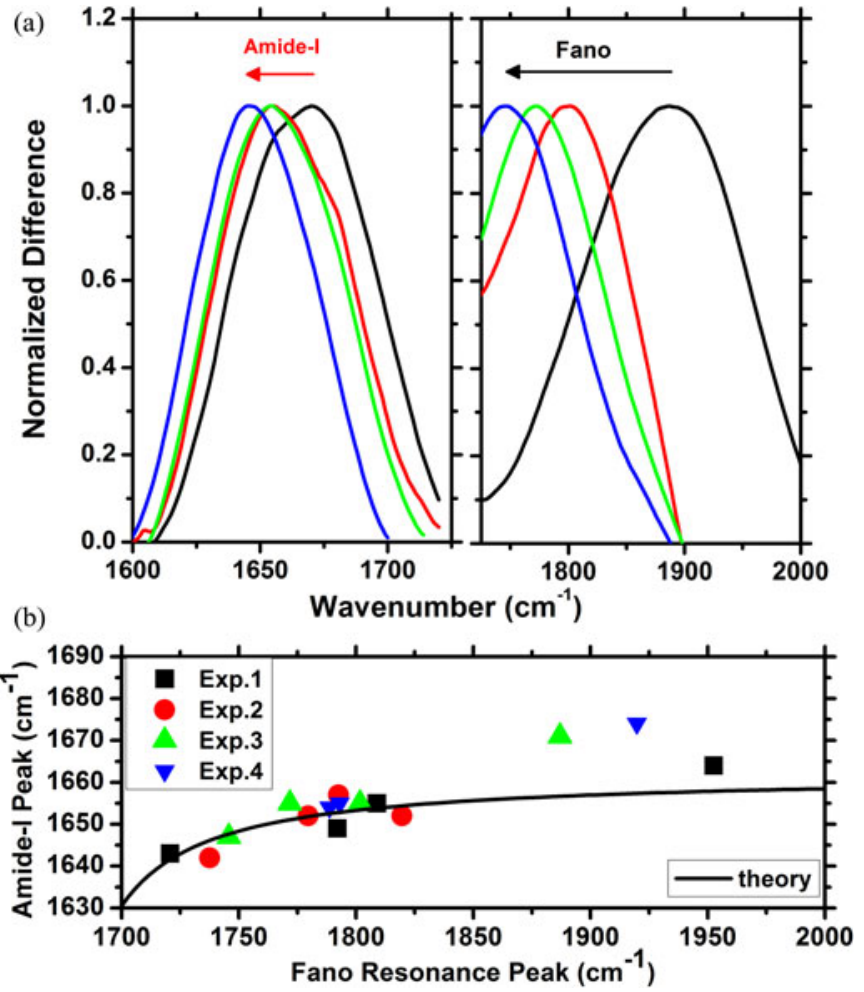

Fig. 4. (a) Normalized experimental difference spectra for baseline corrected Amide-I band and Fano resonance band. (b) Dependence of Amide-I band peak on the Fano-resonance frequency $\kappa=1200 \mathrm{~cm}^{-2}$ and $\omega_{2}=1664 \mathrm{~cm}^{-1}$.

estimate the thickness of the ideal protein monolayer from the magnitude of the normalized difference at the Fano resonance as $5 \mathrm{~nm}$, by using the formula: $\Delta R / R_{Q} \sim h \Delta \varepsilon$. For metamaterials operating at the IR band, by changing the scale factor, we can tune the Fano-resonance frequency to approach the protein vibration modes. In Fig. 3, as the Fano resonance approaches the Amide-I/II lines, the signal magnitude is consistently increasing, which is critical for the detection of proteins and the calculation of their secondary structures [41], [59].

\section{HybridiZATION OF FANO AND AMIDE-I RESONANCES}

By processing the normalized difference spectra, we can precisely locate the position of Amide-I vibrational lines of streptavidin. As the Fano resonance approaches the Amide-I mode, hybridization of Amide-I and Fano resonances leads to a spectral shift of the Amide-I lines as shown in Fig. 4(a). We observed this phenomenon consistently for four different sets of experiments which is the signature of a different Fano resonance between the protein and quadrupole mode of the metamaterial layer. Eigenfrequencies of the new coupled system can be formulated as follows [45]:

$$
\begin{aligned}
\omega_{1,2}^{\prime}= & \frac{\omega_{1}+\omega_{2}}{2} \\
& \quad \pm\left(\frac{\omega_{1}-\omega_{2}}{2}\right)\left[1+\frac{\kappa}{\left(\frac{\omega_{1}-\omega_{2}}{2}\right)^{2}}\right]^{1 / 2} \\
\kappa= & \gamma_{1} \gamma_{2}+\left|\kappa_{12}\right|^{2} .
\end{aligned}
$$

TABLE I

DEMONSTRATIVE PARAMETERS OF HYBRIDIZATION FOR A REPRESENTATIVE DATASET

\begin{tabular}{ccc}
\hline \hline $\begin{array}{c}\text { Fano Resonance } \\
\left(\mathrm{cm}^{-1}\right)\end{array}$ & $\begin{array}{c}\text { Amide-I } \\
\left(\mathrm{cm}^{-1}\right)\end{array}$ & $\begin{array}{c}\text { Estimated } \\
\text { Enhancement }(\%)\end{array}$ \\
\hline 1953 & 1664 & 0.8 \\
1809 & 1655 & 2.8 \\
1792 & 1649 & 3.4 \\
1721 & 1643 & 4.5 \\
\hline \hline
\end{tabular}

Here, $\omega_{1}$ and $\omega_{2}$ represent the metamaterial and Amide-I resonance frequencies for the uncoupled case. The coupling mechanism is described with an overall coupling coefficient: $\kappa$, which depends on the metasurface and Amide-I loss factors, $\gamma_{1}, \gamma_{2}$, and their coupling coefficient $\kappa_{12}$ that is proportional to the index-induced shift $(\Delta \omega)$ obtained from the perturbation theory. In Fig. 4(b), we summarize the results of the experimental sets and compare them with the theoretical model. In order to better understand the magnitude of change in spectra, we tabulate the position of the Fano resonance, the Amide peak, and estimated enhancement in percentage in Table I. Enhancement of the vibrational mode signals is estimated relative to baseline of a normalized reflectance difference dataset. Enhancement can be clearly observed when Fano resonance is nearby the Amide-I vibrational mode and reaches $4.5 \%$. The extracted parameters from this theoretical fit can provide significant information for the mass content and structure of the bound proteins.

\section{CONCLUSION}

To sum up, this technique (Hybrid-SEIRA) allows us to observe the vibrational modes of a bound molecule and how they couple to the underlying metasurface (as the vibrational modes appear at slightly different frequencies on substrates with different Fano resonances). We can also simultaneously monitor the binding of a material to the surface through monitoring the shift of the Fano resonance with changing refractive index. Our ability to gather all of this data on a single sample in an optical, label-free, and nonperturbative manner opens up interesting opportunities for future research with this technique. For example, the technique could be applied to study protein conformational changes as a function of surface packing density, or ordering in polymer films. Since the hybridization between vibrational modes and the Fano resonance enhances the apparent size of spectral red shifts slightly red of the Fano resonance (or blue shifts slightly blue of the Fano resonance), this technique will be especially sensitive at detecting minute changes in vibrational energies.

\section{ACKNOWLEDGMENT}

The author would like to thank K. M. Mayer for the synthesis of proteins on metamaterial substrates and for fruitful discussions. He would also like to thank D. Fozdar, R. Garcia, J. Bolinger, G. Shvets, K. Willets, A. Alu, and X. Xiang from The University of Texas at Austin and TUBITAK 2232 program. 


\section{REFERENCES}

[1] J. C. Venter et al., "The sequence of the human genome," Science, vol. 291, no. 5507, pp. 1304-1351, Feb. 2001.

[2] L. S. Jung, K. E. Nelson, P. S. Stayton, and C. T. Campbell, "Binding and dissociation kinetics of wild-type and mutant streptavidins on mixed biotin-containing alkylthiolate monolayers," Langmuir, vol. 16, no. 24, pp. 9421-9432, Nov. 2000.

[3] M. Mrksich, J. R. Grunwell, and G. M. Whitesides, "Biospecific adsorption of carbonic anhydrase to self-assembled monolayers of alkanethiolates that present benzenesulfonamide groups on gold," J. Amer. Chem. Soc., vol. 117, pp. 12009-12010, 1995.

[4] N. M. Green, "Avidin,” Adv. Protein Chem., vol. 29, pp. 85-133, 1975.

[5] W. Knoll, M. Zizlsperger, T. Liebermann, S. Arnold, A. Badia, M. Liley, D. Piscevic, F. J. Schmitt, and J. Spinke, "Streptavidin arrays as supramolecular architectures in surface-plasmon optical sensor formats," Colloids Surfaces A: Physicochem. Eng. Aspects, vol. 161, no. 1, pp. 115137, Jan. 2000.

[6] A. J. Haes and R. P. Van Duyne, "A nanoscale optical biosensor: Sensitivity and selectivity of an approach based on the localized surface plasmon resonance spectroscopy of triangular silver nanoparticles," J. Amer. Chem. Soc., vol. 124, no. 35, pp. 10596-10604, Sep. 2002.

[7] K. E. Nelson, L. Gamble, L. S. Jung, M. S. Boeckl, E. Naeemi, S. L. Golledge, T. Sasaki, D. G. Castner, C. T. Campbell, and P. S. Stayton, "Surface characterization of mixed self-assembled monolayers designed for streptavidin immobilization," Langmuir, vol. 17, no. 9, pp. 2807-2816, May 2001.

[8] L. Haussling, H. Ringsdorf, F. J. Schmitt, and W. Knoll, "Biotinfunctionalized self-assembled monolayers on gold: Surface plasmon optical studies of specific recognition reactions," Langmuir, vol. 7, no. 9, pp. 1837-1840, Sep. 1991.

[9] T. Liebermann, W. Knoll, P. Sluka, and R. Herrmann, "Complement hybridization from solution to surface-attached probe-oligonucleotides observed by surface-plasmon-field-enhanced fluorescence spectroscopy," Colloids Surfaces A, Physicochem. Eng. Aspects, vol. 169, no. 1-3, pp. 337-350, 2000.

[10] J. S. Shumaker-Parry, M. H. Zareie, R. Aebersold, and C. T. Campbell, "Microspotting streptavidin and double-stranded DNA arrays on gold for high-throughput studies of protein-DNA interactions by surface plasmon resonance microscopy," Anal. Chem., vol. 76, no. 4, pp. 918-929, Feb. 2004.

[11] J. Spinke, M. Liley, F. J. Schmitt, H. J. Guder, L. Angermaier, and W. Knoll, "Molecular recognition at self-assembled monolayers: Optimization of surface functionalization," J. Chem. Phys., vol. 99, no. 9, pp. 7012-7019, 1993.

[12] Z. Yang, W. Frey, T. Oliver, and A. Chilkoti, "Light-activated affinity micropatterning of proteins on self-assembled monolayers on gold," Langmuir, vol. 16, no. 4, pp. 1751-1758, Feb. 1999.

[13] K. M. Mayer, S. Lee, H. Liao, B. C. Rostro, A. Fuentes, P. T. Scully, C. L. Nehl, and J. H. Hafner, "A label-free immunoassay based upon localized surface plasmon resonance of gold nanorods," ACS Nano, vol. 2, no. 4, pp. 687-692, Apr. 2008.

[14] K. M. Mayer, F. Hao, S. Lee, P. Nordlander, and J. H. Hafner, "A single molecule immunoassay by localized surface plasmon resonance," Nanotechnology, vol. 21, no. 25, pp. 255503-1-255503-8, Jun. 2010.

[15] E. Ozbay, "Plasmonics: Merging photonics and electronics at nanoscale dimensions," Science, vol. 311, no. 5758, pp. 189-193, Jan. 2006.

[16] T. Liebermann and W. Knoll, "Surface-plasmon field-enhanced fluorescence spectroscopy," Colloids Surfaces A, Physicochem. Eng. Aspects, vol. 171, no. 1-3, pp. 115-130, Oct. 2000.

[17] K. Kneipp, Y. Wang, H. Kneipp, L. T. Perelman, I. Itzkan, R. Dasari, and M. S. Feld, "Single molecule detection using surface-enhanced Raman scattering (SERS)," Phys. Rev. Lett., vol. 78, no. 9, pp. 1667-1670, Mar. 1997.

[18] C. Genet and T. W. Ebbesen, "Light in tiny holes," Nature, vol. 445, no. 7123, pp. 39-46, Jan. 2007.

[19] S. Lal, N. K. Grady, J. Kundu, C. S. Levin, J. B. Lassiter, and N. J. Halas, "Tailoring plasmonic substrates for surface enhanced spectroscopies," Chem. Soc. Rev., vol. 37, no. 5, pp. 898-911, Apr. 2008.

[20] F. Le, D. W. Brandl, Y. A. Urzhumov, H. Wang, J. Kundu, N. J. Halas, J. Aizpurua, and P. Nordlander, "Metallic nanoparticle arrays: A common substrate for both surface-enhanced Raman scattering and surfaceenhanced infrared absorption," ACS Nano, vol. 2, no. 4, pp. 707-718, Apr. 2008 .
[21] S. A. Love, B. J. Marquis, and C. L. Haynes, "Recent advances in nanomaterial plasmonics: Fundamental studies and applications," Appl. Spectros., vol. 62, no. 12, pp. 346 A-362 A, Dec. 2008.

[22] M. J. Mulvihill, X. Y. Ling, J. Henzie, and P. D. Yang, "Anisotropic etching of silver nanoparticles for plasmonic structures capable of singleparticle SERS," J. Amer. Chem. Soc., vol. 132, no. 1, pp. 268-274, Jan. 2010.

[23] T. Nagao, G. Han, C. Hoang, J. S. Wi, A. Pucci, D. Weber, F. Neubrech, V. M. Silkin, D. Enders, O. Saito, and M. Rana, "Plasmons in nanoscale and atomic-scale systems," Sci. Technol. Adv. Mater, vol. 11, no. 5, Oct. 2010.

[24] R. Adato, A. A. Yanik, J. J. Amsden, D. L. Kaplan, F. G. Omenetto, M. K. Hong, S. Erramilli, and H. Altug, "Ultra-sensitive vibrational spectroscopy of protein monolayers with plasmonic nanoantenna arrays," Proc. Nat. Academy Sci. USA, vol. 106, no. 46, pp. 19227-19232, Nov. 2009.

[25] R. Bukasov and J. S. Shumaker-Parry, "Silver nanocrescents with infrared plasmonic properties as tunable substrates for surface enhanced infrared absorption spectroscopy," Anal. Chem., vol. 81, no. 11, pp. 4531-4535, Jun. 2009.

[26] A. Dhawan, S. J. Norton, M. D. Gerhold, and T. Vo-Dinh, "Comparison of FDTD numerical computations and analytical multipole expansion method for plasmonics-active nanosphere dimers," Opt. Exp., vol. 17, no. 12, pp. 9688-9703, Jun. 2009.

[27] Z. R. Guo, X. Fan, L. K. Liu, Z. P. Bian, C. R. Gu, Y. Zhang, N. Gu, D. Yang, and J. N. Zhang, "Achieving high-purity colloidal gold nanoprisms and their application as biosensing platforms," J. Colloid Interface Sc., vol. 348, no. 1, pp. 29-36, Aug. 2010.

[28] J. Heer, L. Corwin, K. Cilwa, M. A. Malone, and J. V. Coe, "Infrared sensitivity of plasmonic metal films with hole arrays to microspheres in and out of the holes," J. Phys. Chem. C, vol. 114, no. 1, pp. 520-525, Jan. 2010.

[29] D. Enders, T. Nagao, A. Pucci, T. Nakayama, and M. Aono, "Surfaceenhanced ATR-IR spectroscopy with interface-grown plasmonic goldisland films near the percolation threshold," Phys. Chem. Chem. Phys., vol. 13, no. 11, pp. 4935-4941, 2011.

[30] D. Weber, P. Albella, P. Alonso-Gonzalez, F. Neubrech, H. Gui, T. Nagao, R. Hillenbrand, J. Aizpurua, and A. Pucci, "Longitudinal and transverse coupling in infrared gold nanoantenna arrays: Long range versus short range interaction regimes," Opt. Exp., vol. 19, no. 16, pp. 15047-15061, Aug. 2011.

[31] A. Hartstein, J. R. Kirtley, and J. C. Tsang, "Enhancement of the infrared absorption from molecular monolayers with thin metal overlayers," Phys. Rev. Lett., vol. 45, no. 3, pp. 201-204, 1980.

[32] H. Wang, J. Kundu, and N. J. Halas, "Plasmonic nanoshell arrays combine surface-enhanced vibrational spectroscopies on a single substrate," Angewandte Chemie-Int. Edition, vol. 46, no. 47, pp. 9040-9044, 2007.

[33] F. Hao, P. Nordlander, Y. Sonnefraud, P. Van Dorpe, and S. A. Maier, "Tunability of subradiant dipolar and fano-type plasmon resonances in metallic ring/disk cavities: Implications for nanoscale optical sensing," ACS Nano, vol. 3, no. 3, pp. 643-652, Mar. 2009.

[34] P. Alonso-Gonzalez, M. Schnell, P. Sarriugarte, H. Sobhani, C. H. Wu, N. Arju, A. Khanikaev, F. Golmar, P. Albella, L. Arzubiaga, F. Casanova, L. E. Hueso, P. Nordlander, G. Shvets, and R. Hillenbrand, "Real-Space mapping of fano interference in plasmonic metamolecules," Nano Lett., vol. 11, no. 9, pp. 3922-3926, Sep. 2011.

[35] Y. Gu, Q. Z. Li, J. Xiao, K. D. Wu, and G. P. Wang, "Plasmonic metamaterials for ultrasensitive refractive index sensing at near infrared," J. Appl. Phys., vol. 109, no. 2, pp. 023104-1-023104-6, Jan. 2011.

[36] B. Kante, A. de Lustrac, and J. M. Lourtioz, "In-plane coupling and field enhancement in infrared metamaterial surfaces," Phys. Rev. B, vol. 80, no. 3, pp. 035108-1-035108-6, Jul. 2009.

[37] I. M. Pryce, K. Aydin, Y. A. Kelaita, R. M. Briggs, and H. A. Atwater, "Highly strained compliant optical metamaterials with large frequency tunability," Nano Lett., vol. 10, no. 10, pp. 4222-4227, Oct. 2010.

[38] I. M. Pryce, Y. A. Kelaita, K. Aydin, and H. A. Atwater, "Compliant metamaterials for resonantly enhanced infrared absorption spectroscopy and refractive index sensing," ACS Nano, vol. 5, no. 10, pp. 8167-8174, Oct. 2011 .

[39] K. B. Alici, F. Bilotti, L. Vegni, and E. Ozbay, "Optimization and tunability of deep subwavelength resonators for metamaterial applications: complete enhanced transmission through a subwavelength aperture," Opt. Exp., vol. 17, no. 8, pp. 5933-5943, Apr. 2009.

[40] K. B. Alici, A. E. Serebryannikov, and E. Ozbay, "Radiation properties and coupling analysis of a metamaterial based, dual polarization, dual 
band, multiple split ring resonator antenna," J. Electromagn. Waves Appl., vol. 24, no. 8-9, pp. 1183-1193, 2010.

[41] E. Cubukcu, S. Zhang, Y.-S. Park, G. Bartal, and X. Zhang, "Split-ring resonator sensors for infrared detection of single molecular monolayers," Appl. Phys. Lett., vol. 95, no. 4, pp. 043113-1-043113-3, Jul. 2009.

[42] F. Neubrech, A. Pucci, T. W. Cornelius, S. Karim, A. Garcia- Etxarri, and J. Aizpurua, "Resonant plasmonic and vibrational coupling in a tailored nanoantenna for infrared detection," Phys. Rev. Lett., vol. 101, no. 15, pp. 157403-1-157403-4, Oct. 2008.

[43] B. Luk'yanchuk, N. I. Zheludev, S. A. Maier, N. J. Halas, P. Nordlander, H. Giessen, and C. T. Chong, "The Fano resonance in plasmonic nanostructures and metamaterials," Nat. Mater., vol. 9, no. 9, pp. 707-715, Sep. 2010.

[44] C. H. Wu, A. B. Khanikaev, R. Adato, N. Arju, A. A. Yanik, H. Altug, and G. Shvets, "Fano-resonant asymmetric metamaterials for ultrasensitive spectroscopy and identification of molecular monolayers," Nat. Mater. vol. 11, no. 1, pp. 69-75, Jan. 2012.

[45] H. A. Haus and W. P. Huang, "Coupled-Mode theory," Proc. IEEE, vol. 79, no. 10, pp. 1505-1518, Oct. 1991.

[46] R. E. Hamam, A. Karalis, J. D. Joannopoulos, and M. Soljacic, "Coupledmode theory for general free-space resonant scattering of waves," Phys. Rev. A, vol. 75, no. 5, pp. 053801-1-053801-5, May 2007.

[47] Z. C. Ruan and S. H. Fan, "Temporal coupled-mode theory for fano resonance in light scattering by a single obstacle," J. Phys. Chem. C, vol. 114, no. 16, pp. 7324-7329, Apr. 2010.

[48] B. M. W. Trapnell, "The activities of evaporated metal films in gas chemisorption," Proc. Roy. Soc. London. A, Math. Phys. Sci., vol. 218, no. 1135 , pp. 566-577, Jul. 1953.

[49] S. D. Evans, R. Sharma, and A. Ulman, "Contact angle stability: Reorganization of monolayer surfaces?" Langmuir, vol. 7, no. 1, pp. 156-161, Jan. 1991.

[50] L. M. Fischer, M. Tenje, A. R. Heiskanen, N. Masuda, J. Castillo, A. Bentien, J. Émneus, M. H. Jakobsen, and A. Boisen, "Gold cleaning methods for electrochemical detection applications," Microelectronic Eng., vol. 86, no. 4-6, pp. 1282-1285.

[51] K. K. Caswell, J. N. Wilson, U. H. F. Bunz, and C. J. Murphy, "Preferential end-to-end assembly of gold nanorods by biotin-streptavidin connectors," J. Am. Chem. Soc., vol. 125, no. 46, pp. 13914-13915, Nov. 2003.

[52] I. Le Trong, Z. Wang, D. E. Hyre, T. P. Lybrand, P. S. Stayton, and R. E. Stenkamp, "Streptavidin and its biotin complex at atomic resolution," Acta Crystallographica Section D-Biological Crystallography, vol. 67, pp. 813-821, Sep. 2011.

[53] G. U. Lee, D. A. Kidwell, and R. J. Colton, "Sensing discrete streptavidinbiotin interactions with atomic force microscopy," Langmuir, vol. 10, no. 2, pp. 354-357, Feb. 1994.

[54] W. A. Hendrickson, A. Pahler, J. L. Smith, Y. Satow, E. A. Merritt, and R. P. Phizackerley, "Crystal structure of core streptavidin determined from multiwavelength anomalous diffraction of synchrotron radiation," Proc. Nat. Academy Sci. USA, vol. 86, no. 7, pp. 2190-2194, Apr. 1989.
[55] J. B. Lee, S. H. Um, J.-W. Choi, and K.-K. Koo, "Elimination of aggregates of ferredoxin from its self-assembled monolayer on silicon substrate," Colloids Surfaces B, Biointerfaces, vol. 30, no. 4, pp. 307-314, 2003.

[56] T. M. McIntire, A. Scott Lea, D. J. Gaspar, N. Jaitly, Y. Dubowski, Q. Li, and B. J. Finlayson-Pitts, "Unusual aggregates from the oxidation of alkene self-assembled monolayers: A previously unrecognized mechanism for SAM ozonolysis?" Phys. Chem. Chem. Phys., vol. 7, no. 20 , pp. 3605-3609, 2005.

[57] A. F. Raigoza, D. A. Villalba, N. A. Kautz, and S. A. Kandel, "Structure and self-assembly of sequentially adsorbed coronene/octanethiol monolayers," Surface Sci., vol. 604, no. 19-20, pp. 1584-1590, Sep. 2010.

[58] S. G. Johnson, M. L. Povinelli, M. Soljačić, A. Karalis, S. Jacobs, and J. D. Joannopoulos, "Roughness losses and volume-current methods in photonic-crystal waveguides," Appl. Phys. B, vol. 81, no. 2-3, pp. $283-$ 293, Jul. 2005.

[59] A. Dong, P. Huang, and W. S. Caughey, "Protein secondary structures in water from second-derivative amide I infrared spectra," Biochemistry, vol. 29, no. 13, pp. 3303-3308, Apr. 1990.

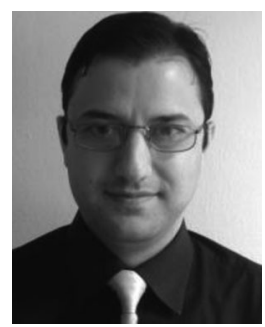

Kamil Boratay Alici (M'13) was born in Sivas, Turkey, on January 12, 1981. He received the B.S. and Ph.D. degrees in physics from Bilkent University, Ankara, Turkey in 2004 and 2010, respectively.

He held permanent Researcher positions in the Department of Electrical Engineering, University of Arkansas, and Department of Physics, University of Texas at Austin, from 2010 to 2013. He is currently a Research Assistant Professor in Nanotechnology Research Center, Bilkent University, Ankara, Turkey. His scientific interests include applications of nanophotonics in sustainable energy, life sciences, and communication technologies. He has introduced optically thin broadband near infrared absorbers/emitters, electrically small RF and optical antennas, ultrathin microwave absorbers, negative index materials and superlenses at millimeter wave band, negative refraction in photonic crystals, hybrid sensing platforms for biomolecules, graphene nanophotonics; low cost, high efficiency a-Si based solar cells that are incorporated with spectral energy up-converters, unusual laser damage threshold (LDT) of metasurfaces, and he has contributed to SiC-based compact particle accelerator/detector project. $\mathrm{He}$ is the author of more than 30 journal papers listed in science citation index with $750+$ citations and an h-index of 15 .

Dr. Alıcı received the Undergraduate Scholarship (2001-2004), and Graduate Scholarship (2004-2009) of the Scientific and Technological Research Council of Turkey. 